\title{
Arm pain after syncope: Diagnosis
}

\section{S K Misser, MB ChB, FCRad (D)}

Lake Smit and Partners, Durban

Corresponding author: S Misser (shalendramisser@hotmail.com)
Please refer to page 63 of the June 2011 issue of the SAJR (http://www. sajr.org.za/index.php/sajr/article/view/579/443) for the presentation details. We congratulate Dr Himal Gajjar (Schnetler, Corbett and Partners, Cape Town) for a precise and detailed diagnosis, for which he receives an award of R1 000 from the RSSA. Dr Misser elaborates below on the condition and its imaging.

\section{Presentation and diagnosis}

(Due to space constraints, the images referred to below are not reproduced here but are in the online June 2011 SAJR at the above URL.)

The patient presented with arm pain after a syncopal episode. She had encountered numerous episodes of dizziness and falls that were attributed to her long-standing renal failure. However, the patient reported that, despite routine dialysis, she had felt a difference with generalised weakness and fatigue becoming progressively worse. The initial series of plain radiographs performed on presentation demonstrate (in Fig. 1) the dialysis catheter in position and 'rugger jersey' appearance of the dorsal spine. Fig. 2 shows focal medial humeral neck sub-periosteal resorption with an undisplaced fracture. There are also lucent foci of varying sizes with endosteal scalloping owing to multiple brown tumours. A remodelled right fifth rib probably is a healed fracture. Fig. 3 reveals marked endosteal and trabecular resorption of tibia and fibula as well as distal third fibular periostitis change. Prompted by these radiographic features of renal osteodystrophy and secondary hyperparathyroidism, a series of blood tests were performed. These confirmed low serum calcium, hyperphosphataemia and markedly raised parathormone $(1617 \mathrm{pg} / \mathrm{ml})$.

Abdominal radiographs (Fig. 4) taken 6 weeks later show that a new catheter has been placed via the left femoral vein, with its tip in the IVC. Bony changes with demineralisation, brown cysts and vertebral end plate sclerosis are noted. Fig. 5 demonstrates a solitary uncomplicated gallbladder calculus, a not uncommon finding in hyperparathyroidism.

This case is another example where the radiologist may offer a definitive diagnosis (renal osteodystrophy with secondary hyperparathyroidism) based on diagnostic radiographic features aiding further clinical management.

\section{Discussion}

Of all endocrine organs, the parathyroid gland is unique, being the only one not under the control of the hypothalamic-pituitary axis. The parathyroid gland secretes parathormone (PTH) on the basis of serum calcium and phosphate levels. In renal failure, phosphorus retention stimulates increased PTH production and vitamin D is not converted to its active form. Both occur due to a complex cascade involving FGF-23 with reduction in phosphaturia and inhibition of 1-a hydroxylase.

Clinically, hyperparathyroidism presents with 'bones, stones, groans and moans', i.e. manifesting by bone pain, kidney/gallstones, gastrointestinal symptoms and lethargy/weakness respectively.

There are numerous radiographic features of hyperparathyroidism (see Table I). A sine qua non for hyperparathyroidism is subperiosteal resorption along the radial side of middle phalanges. ${ }^{1}$ Vertebral end plate osteosclerosis is particularly specific for secondary hyperparathyroidism. ${ }^{2}$ Renal osteodystrophy, as in the patient presented here, is the term used to describe the combination of renal dysfunction coupled with hyperparathyroidism and the associated soft tissue and skeletal changes. ${ }^{3}$

1. Helms, CA. Fundamentals of Skeletal Radiology, 3rd ed. Philadelphia, USA: Saunders, 2005:145-147.

2. Lenchik L, Mitchell K. Hyperparathyroidism and Renal Osteodystrophy. In: El-Khoury G, ed. Essentials of Musculoskeletal Imaging. Philadelphia, USA: Churchill Livingstone, 2003:295-301.

3. Resnick D. Chapter 52: Parathyroid Disorders and Renal Osteodystrophy. In: Diagnosis of Bone and Joint Disorders, 4th ed. Philadelphia, USA: Saunders, 2002:2043-2111.

\section{Erratum}

The award winner of the new quiz case in the March 2011 issue of the SAJR was incorrectly announced as Dr Himal Gajjar of Schnetler, Corbett and Partners, Cape Town. In fact, the winner is Dr M Omar, a radiology consultant at Chris Hani Baragwanath Hospital. However, as it turns out, Dr Gajjar is the winner of the quiz case in the June 2011 issue. We congratulate both winners, and apologise for the error.

Table I. Radiographic features of hyperparathyroidism and common sites at which they occur

Radiographic feature
1. Bone resorption (osteopaenia)
- Sub-periosteal
- Intracortical
- Endosteal
- Trabecular
- Sub-ligamentous
- Sub-chondral
2. Bone sclerosis
3. Brown tumours
4. Periostitis
5. Stones

\section{Common sites}

Radial aspect of middle phalanges, medial side of humerus, tibia

Terminal tufts(acro-osteolysis)

Hands and long bones

Skull (salt and pepper appearance)

Trochanters, calcaneus, ischial/humeral tuberosities, lateral clavicle

Acromioclavicular, sacro-iliac and sternoclavicular joints

Rugger jersey spine, cranio-facial bony hypertrophy with leonine facies

Mandible, clavicle, ribs, pelvis, long bones and hands

Long bones, esp. tibia, fibula, femur

Gallstones, kidney stones or nephrocalcinosis 